\title{
TAXonomía de SPERMACOCE ERYNgIOIDES (RubiaceAe) Y transferencia de BorReria secC. Pseudodiodia a SPERMacoce
}

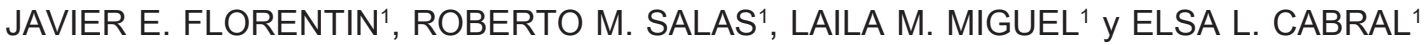

\begin{abstract}
Resumen: Spermacoce eryngioides fue considerada en los últimos 20 años como una especie del género Borreria con las variedades affinis, eryngioides y ostenii. En este trabajo se analizaron microcaracteres florales, carpológicos, seminales y polínicos, y aspectos ecológicos y distribucionales, a fin de evaluar el status taxonómico de las tres variedades. Por otro lado, se analizó la situación taxonómica de Borreria sección Pseudodiodia. Como resultado dos variedades de S. eryngioides se elevan al rango de especie como S. hassleri y S. ostenii. Además se considera a Borreria sección Pseudodiodia como nuevo sinónimo del género Spermacoce, se incluye una lista de 16 especies transferidas a Spermacoce y se designa un lectotipo para el nombre $S$. suberecta.
\end{abstract}

Palabras clave: Borreria sección Pseudodiodia, Spermacoce hassleri, S. ostenii.

\begin{abstract}
Summary: Taxonomy of Spermacoce eryngioides (Rubiaceae) and transference of Borreria section Pseudodiodia to Spermacoce. Spermacoce eryngioides was considered in the last 20 years as part of genus Borreria with the varieties affinis, eryngioides, and ostenii. In this work, floral, carpological, seminal and pollen microcharacters were analyzed, and ecological and distributional features were performed to assess the taxonomic status of these three varieties. On the other hand, the taxonomical situation of Borreria section Pseudodiodia was analyzed. As a result, two varieties of $S$. eryngioides were elevated to species level as S. hassleri and S. ostenii. In addition, Borreria section Pseudodiodia was considered as a new synonym of genus Spermacoce, a list of 16 transferred species to Spermacoce was included and a lectotype for the name $S$. suberecta was designated.
\end{abstract}

Key words: Borreria section Pseudodiodia, Spermacoce hassleri, S. ostenii.

\section{INTRODUCCIÓN}

El género Spermacoce L. pertenece a la tribu Spermacoceae (Rubiaceae) y dentro de ésta se encuentra actualmente ubicado en el clado "Spermacoce" (Karehed et al., 2008). Consta de especies distribuidas en los trópicos y subtrópicos de América, África, Asia y Australia (Salas et al., 2015). Durante los últimos dos siglos, los límites del género fueron razón de intensos debates, los cuales se consideran aún irresueltos (Salas et al., 2011). En este sentido numerosas especies fueron transferidas a Spermacoce provenientes de géneros afines como

\footnotetext{
${ }^{1}$ Facultad de Ciencias Exactas, Naturales y Agrimensura. Instituto de Botánica del Nordeste (UNNE-CONICET), Sargento Cabral 2131, c.c. 209, CP 3400. FACENAUNNE. Corrientes, Argentina. E-mail: florentinjaviere@ gmail.com
}

Borreria G. Mey., Diodia L. y Galianthe Griseb. (Govaerts, 1996; Delprete, 2007; Salas et al., 2011), lo cual hace difícil precisar el número de taxones. De acuerdo a las diferentes posturas, las especies de Spermacoce varían de tres en los conceptos más restrictivos (Bacigalupo, 1972) a más de 250 en los más amplios (Govaerts, 1996). Si bien los límites del género no están del todo claros, Cabral et al. (2010) propusieron incluir en este género a especies con las siguientes características similares a la especie tipo, Spermacoce tenuior L.: inflorescencias pseudoaxilares (con desarrollo unilateral), estigma incluso, estambres fijos en la base o en la mitad del tubo corolino y granos de polen subprolatos o prolato-esferoidales, con colpos largos, exina tectada y con espínulas distribuidas alrededor de la ectoabertura (Tipo I según Pire, 1996; Dessein et al., 2002), fruto 2-carpelar, indehiscente o parcialmente dehiscente con un carpelo con dehiscencia apical 
septicida y el otro indehiscente. Las autoras incluyeron en este concepto, Spermacoce s. str., a siete especies (i.e., Spermacoce confusa Rendle, $S$. exasperata Urb., S. glabra Michx., S. keyensis Small, S. pilifera Bacigalupo, S. riparia Cham. \& Schltdl. y S. tetraquetra A. Rich.), además de la especie tipo.

Por otro lado, dentro del género morfológicamente afín Borreria existe un grupo de especies ubicadas por Bacigalupo \& Cabral (1996) dentro de Borreria secc. Pseudodiodia Hassl., con características morfológicas muy similares a Spermacoce. En dicho trabajo, las autoras definieron al taxón por tener estigma incluso, estambres fijos en la base o en la mitad del tubo corolino y el mismo tipo polínico (Tipo I), con la diferencia en la dehiscencia del fruto, el cual tiene ambas valvas dehiscentes (Cabral \& Bacigalupo, 1996). Debido que esa es la única diferencia, las especies incluidas en esta sección fueron recientemente consideradas todas dentro del género Spermacoce (Cabral \& Salas, 2015). Las restantes especies permanecen en el género Borreria y se diferencian por tener inflorescencias apicales y axilares rodeando el eje florífero, estambres y estigmas notoriamente exertos, estambres fijos en el seno interlobular, y granos de polen multiaperturados, pero nunca como lo descrito para este grupo tratado (para una descripción completa ver Pire, 1996). Entre las especies de Borreria secc. Pseudodiodia, B. eryngioides Cham. $\&$ Schltdl. es la única que presenta dos variedades además de la típica, $B$. eryngioides var. affinis (DC.) K. Schum. y B. eryngioides var. ostenii (Standl.) E. L. Cabral \& Bacigalupo. Sin embargo, dichas variedades fueron provisionalmente consideradas como parte de una única especie relativamente polimórfica denominada Spermacoce eryngioides (Cham. \& Schltdl.) Kuntze (Cabral \& Salas, 2015). Como parte de los estudios de las Rubiáceas para la flora Argentina, se analizaron detalladamente las variedades de este taxón teniendo en cuenta caracteres exomorfológicos, microcaracteres florales, polínicos, seminales y corológicos a fin de evaluar el status taxonómico de las mismas. Se analiza además la situación taxonómica de Borreria secc. Pseudodiodia.

\section{Materiales y Métodos}

Para el tratamiento taxonómico se analizaron los ejemplares tipos de todas las especies y materiales representativos depositados en los siguientes herbarios: BR, CGE, CTES, CORD, CRC, E, ESA, F, G, G-DC, HAL, HUEFS, K, L, LIL, MBM, MO, NY, P, R, SI, UB, UEC, y US (acrónimos según Thiers et al., 2015). También se consultaron imágenes de los tipos de alta resolución disponibles en Jstor@ (http://plants.jstor.org/).

Para el análisis de microcaracteres morfológicos se utilizó material fijado en FAA, secado a punto crítico y metalizado con Oro-Paladio y se observó en el Microscopio Electrónico de Barrido (MEB) Jeol LV 5800 del Servicio de Microscopía Electrónica de la UNNE, Corrientes. Las observaciones se realizaron con lupa estereoscópica (LE) y microscopio óptico (MO). La morfología polínica se analizó a partir de botones florales extraídos de ejemplares de herbario procesados siguiendo la técnica de acetólisis de Erdtman (1966), y se utilizó gelatina-glicerinada como medio de montaje para la observación con MO. Para cada especie se midió un mínimo de 20 granos y se siguió la terminología de Punt et al. (2007). Los especímenes utilizados en microscopia electrónica para estudios polínicos y micromorfológicos provienen de Argentina y se detallan a continuación: Florentín \& Salas 9 (CTES); Florentín \& Arana 10 (CTES); Judkevich \& Salas 57 (CTES). El estudio de distribución geográfica se basó en más 200 entradas provenientes de materiales de herbario. Para graficar se utilizó el software DIVA-Gis versión 4 (Hijmans, 2013).

\section{Resultados}

En el presente trabajo se incluye nueva información basada en el análisis de colecciones recientes y de observaciones a campo de Spermacoce eryngioides y sus taxones infraespecíficos. Como resultado de este análisis, tres diferentes entidades a nivel de especie pueden ser reconocidas, a saber: Spermacoce eryngioides, S. hassleri E. L. Cabral \& J. Florentín y S. ostenii (Standl.) E. L. Cabral \& J. Florentín. Comentarios comparativos sobre las tres especies y con taxones afines se exponen a continuación.

\section{Corología}

Las tres especies son taxones eminentemente chaqueños debido a que su distribución geográfica (Fig. 1) se encuentran íntegramente en la 


\section{J. E. Florentín et al. - Taxonomia de Spermacoce (Rubiaceae)}

Subregión Chaqueña, provincia biogeográfica del Chaco (Morrone, 2014). En cuanto a hábitat y ecología, Spermacoce eryngioides se encuentra frecuentemente en suelos modificados, arenosos o compactados, bordes de camino, incluso en periferias de cultivos, y prefiere exposición directa al sol. Habita entre los 50-250 m, principalmente en bosques de Schinopsis balansae Engl. y espinillares de Prosopis affinis Spreng., mientras que Spermacoce ostenii, es endémica de las sierras de Córdoba y San Luis entre 800-1300 m. Su área de distribución es restringida a afloramientos rocosos, pertenecientes al Chaco Serrano, crece entre rocas, con suelos arenoso-rocosos algo friables, incluso como pionera en áreas degradas y expuestas. Spermacoce eryngioides y S. hassleri comparten la misma distribución, a excepción de algunas localidades en donde sólo habita hasta el presente la segunda especie, por ejemplo en las provincias de Catamarca, Jujuy, Santiago del Estero (Argentina), o en el departamento de Beni (Bolivia). A pesar de eso, tienen diferencias marcadas en sus microhábitats. A diferencia de Spermacoce eryngioides, S. hassleri es una especie de lugares sombreados o semisombra de ambientes inundables o próximos a cursos de ríos, sobre todo borde o interior de bosques higrófilos y bosques en galería, siguiendo los cursos de los principales ríos (Paraná, Bermejo, Pilcomayo, Paraguay, entre otros) y sus afluentes (Fig. 1).

\section{Hábito, hoja e inflorescencia}

Las tres especies son plantas perennes que difieren principalmente en la altura, forma de crecimiento y ramificaciones de la inflorescencia (Fig. 2 A-B, D-E, G-H). Spermacoce eryngioides y $S$. ostenii son sufrútices erectos o decumbentes que varían entre 10-30 $\mathrm{cm}$ de altura (Fig. $2 \mathrm{D}, \mathrm{G}$ ), mientras que $S$. hassleri alcanza hasta $1 \mathrm{~m}$, con ramas a veces apoyantes. Las hojas en $S$. eryngioides son generalmente lineares o angostamente elípticas, glabras y con nervios incospicuos, características que comparte frecuentemente con S. ostenii (Fig. 2 F, I). Por otro lado, S. hassleri tiene hojas

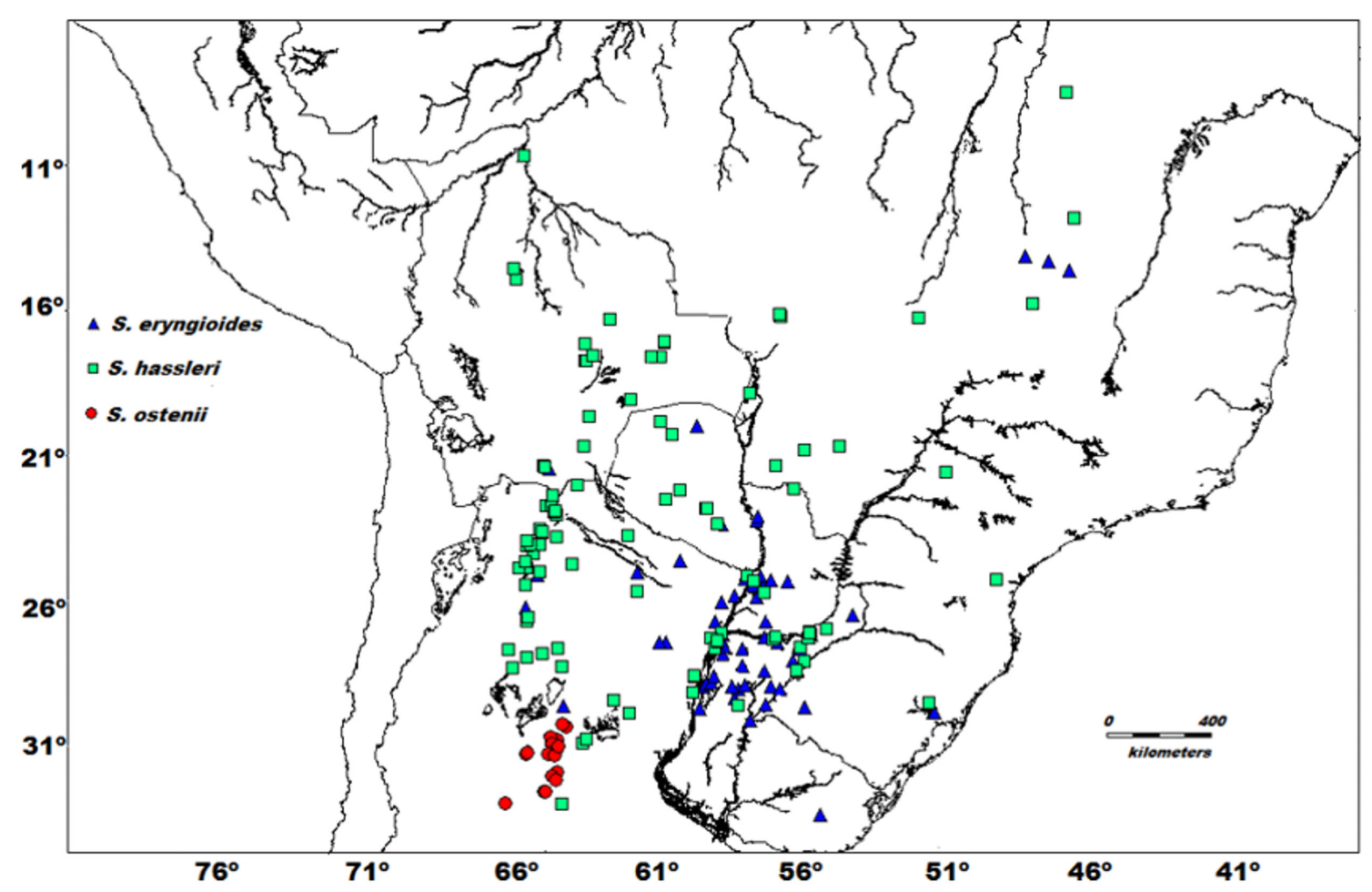

Fig. 1. Distribución de Spermacoce eryngioides, S. hassleri y S. ostenii en Sudamérica. 
Bol. Soc. Argent. Bot. 51 (3) 2016

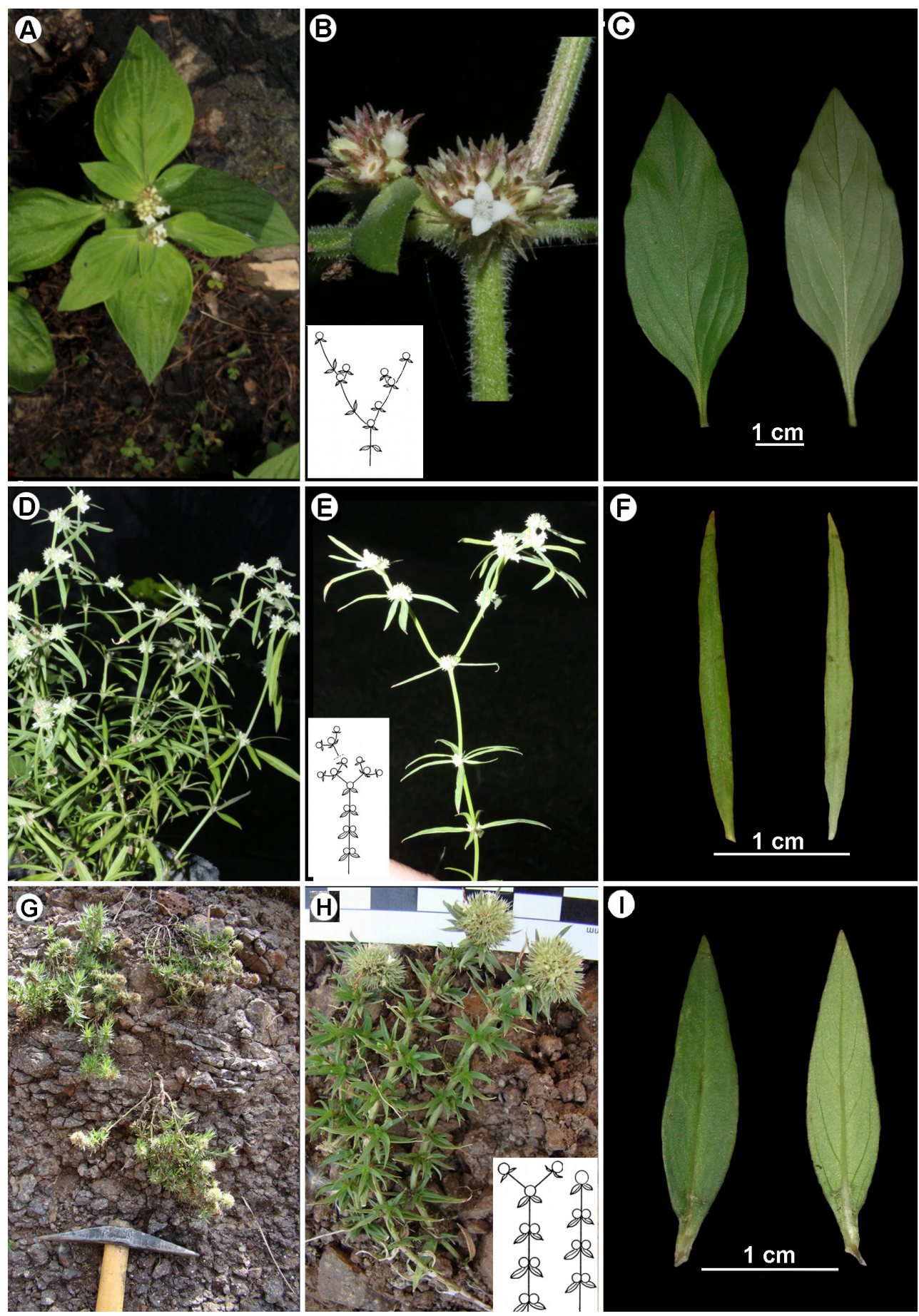

Fig. 2. A-C. Spermacoce hassleri. A. Hábito. B. Inflorescencia pseudodicotómica y esquema de inflorescencia. C. Hojas (vista adaxial y abaxial). S. eryngioides. D. Hábito. E. Inflorescencia dicotómica y esquema de inflorescencia. F. Hojas (vista adaxial y abaxial). G-I. S. ostenii. G. Hábito. H. Inflorescencia en eje florífero simple y esquemas de inflorescencias. I. Hojas (vista adaxial y abaxial). A-C: Florentín \& Salas 9 (CTES); D-F: Judkevich \& Salas 57 (CTES); G-I: Florentín \& Arana 10 (CTES). 


\section{J. E. Florentín et al. - Taxonomia de Spermacoce (Rubiaceae)}

oblongas o elípticas, cartáceas o subcartáceas y pubescentes en ambas caras, con 5-6 nervios secundarios conspicuos (Fig. 2 C). En cuanto a la inflorescencia, Spermacoce ostenii tiene el eje simple (Fig. $2 \mathrm{H}$ ) o con una única ramificación apical dicotómica, mientras que $S$. hassleri y $S$. eryngioides tienen inflorescencias ampliamente ramificadas, en $S$. eryngioides la inflorescencia se divide dicotómicamente hasta cinco veces, es decir que ambas ramas se dividen sucesivamente en dos equivalentes, de manera que se produce una horcadura de ramas iguales (Fig. 2 E). Si bien en $S$. hassleri, el eje se divide también varias veces, éste lo hace siguiendo un patrón pseudo-dicotómico, correspondiendo a una "falsa dicotomía", donde una rama se desarrolla notoriamente más que la otra (Fig. 2 B).

\section{Micromorfología floral}

Las principales diferencias en los análisis de microcaracteres se encontraron en el indumento interno de la corola, la forma del disco nectarífero y la relación de altura del estilo-estigma con respecto al disco (Fig. 3 A-I). En Spermacoce eryngioides los pelos forman una franja densa en la mitad inferior de los lóbulos, desde allí los pelos se distribuyen en forma linear siguiendo la vena media de cada lóbulo hasta la base del tubo y allí se forma un segundo anillo algo más laxo (Fig. $3 \mathrm{~B}, \mathrm{E}$ ); dichos pelos internos miden ca. $320 \mu \mathrm{m}$ de largo. Spermacoce ostenii coincide en el mismo patrón de disposición de pelos, sin embargo la densidad disminuye hacia la base y los pelos son levemente más largos, ca. $400 \mu \mathrm{m}$ de largo (Fig. 3 C, F). En S. hassleri, los pelos de los lóbulos se disponen en un anillo laxo en la mitad inferior de los lóbulos, y continúan hacia el tubo corolino siguiendo la línea de la vena media. A diferencia de las otras dos especies en la base no se observa un segundo anillo (Fig. $3 \mathrm{~A}, \mathrm{D})$, los pelos son en promedio más cortos (ca. $300 \mu \mathrm{m}$ de largo) y se disponen más laxamente. Spermacoce eryngioides tiene disco entero con papilas subcilíndricas (Fig. $3 \mathrm{~K}$ ), S. ostenii bilobado con papilas cónicas (Fig. 3 L) y $S$. hassleri bipartido con papilas subcilíndricas a cónicas (Fig. 3 J). En cuanto al estilo, la última especie tiene más o menos la misma altura que el disco (Fig. $3 \mathrm{G}$ ), sin embargo en las dos restantes los estilos sobrepasan al disco, siendo en S. eryngioides levemente más largo (Fig. $3 \mathrm{H}-\mathrm{I})$.

\section{Fruto y semilla}

En las tres especies, el fruto tiene el mismo patrón de dehiscencia con ambos carpelos dehiscentes. En la semilla, las tres entidades tienen la cara dorsal con exotesta retículo-foveada, con células poligonales de paredes anticlinales rectas y periclinales cóncavas (Fig. 4 A, C, E). En $S$. hassleri y $S$. eryngioides, las células son levemente mayores, ca. 44 y $43 \mu \mathrm{m}$ lat. respectivamente, mientras que en $S$. ostenii ca. $35 \mu \mathrm{m}$ lat. La cara ventral en $S$. eryngioides se diferencia por tener un surco longitudinal angosto y escasamente excavado (Fig. 4 C, derecha), mientras en $S$. hassleri es amplio aunque delimitado por los bordes laterales (Fig. 4 A, derecha) y en $S$. ostenii la cara es totalmente plana, sin surcos notorios (Fig. 4 E, derecha).

\section{Granos de polen}

Los granos de polen resultaron notoriamente homogéneos, en los tres casos son isopolares, radiosimétricos, 3-4-zonocolpados, pequeños, subprolatos, endoabertura en endocíngulo, con exina tectada-psilada y con nanoespinas alrededor de los colpos (Fig. 3 B, D, F). La uniformidad en la morfología polínica se considera como una sinapomorfía para el género, ya que es relativamente bien conservada. Por otro lado, si bien no permite diferenciar entre estas especies, soporta la reubicación de las tres especies en Spermacoce.

\section{Tratamiento taxonómico}

Spermacoce L., Sp. Pl. 102. 1753. Especie Tipo: Spermacoce tenuior L.

Borreria secc. Pseudodiodia Hassl., Repert. Spec. Nov. Regni Veg. 14: 166-167. 1915, nov. syn. Lectotipo (designado por Cabral \& Bacigalupo, 1996): Borreria chacoensis Hassl. (= Spermacoce hassleri E. L. Cabral \& J. Florentín).

El género Spermacoce en América comprende ca. 30 especies distribuidas desde el NE de Estados Unidos hasta el centro de Argentina. En Argentina viven seis especies de las cuales $S$. ostenii es la única endémica y habita en las sierras de Córdoba y San Luis. Varios autores (e. g., Govaerts, 1996; Delprete, 2007) asimilaron todo el género Borreria bajo la sinonimia de Spermacoce, sin embargo, en el presente trabajo sólo se considera que Borreria secc. Pseudodiodia es sinónimo de este género. 
Bol. Soc. Argent. Bot. 51 (3) 2016

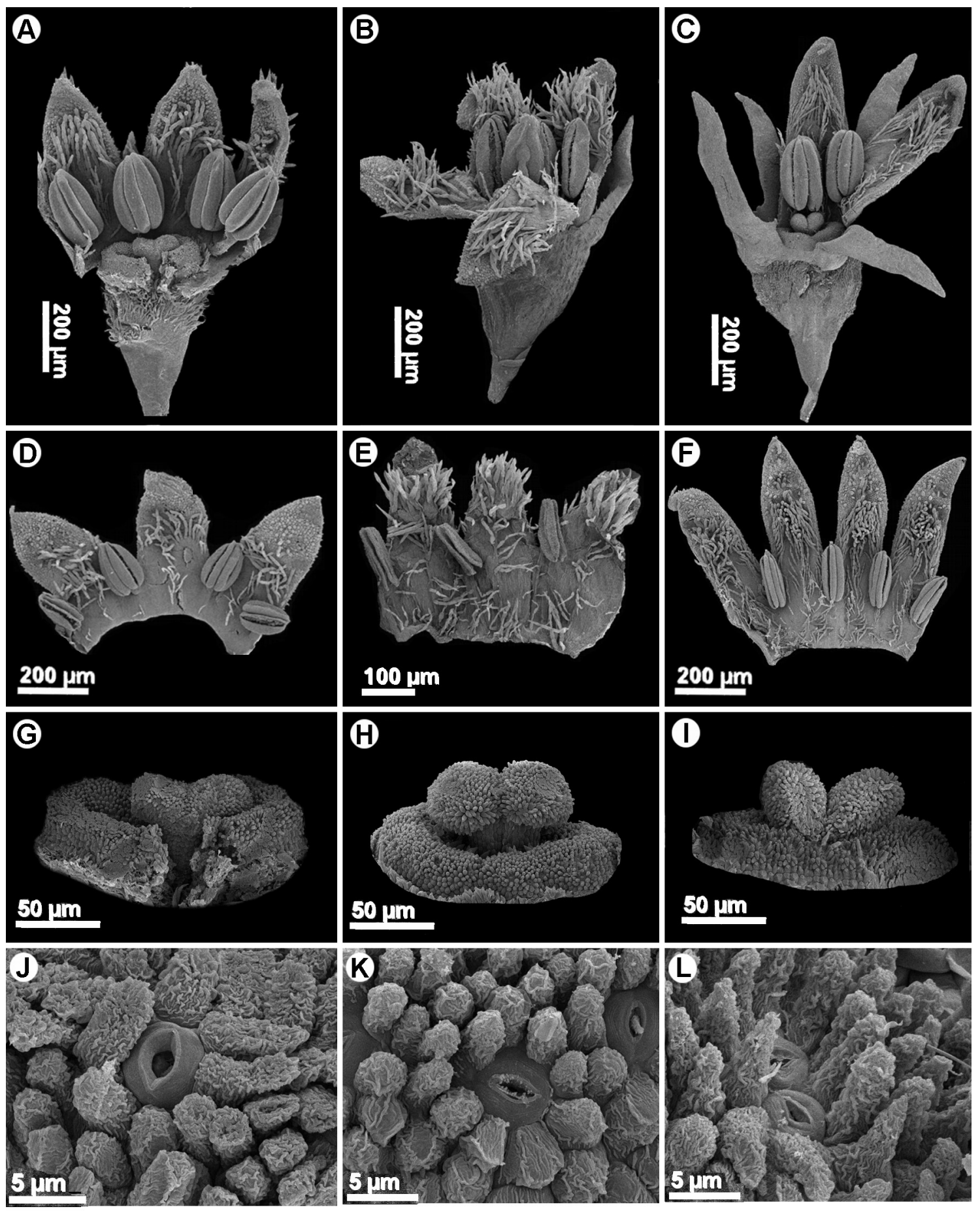

Fig. 3. A, D, G, J. Spermacoce hassleri. A. Flor. D. Corola desplegada. G. Disco bipartido. J. Papilas del disco. B, E, H, K. S. eryngioides. B. Flor. E. Corola desplegada. H. Disco entero. K. Papilas del disco. C, F, I, L. S. ostenii. C. Flor. F. Corola desplegada. I. Disco bilobado. L. Papilas del disco. A, D, G, J: Florentín \& Salas 9 (CTES); B, E, H, K: Judkevich \& Salas 57 (CTES); C, F, I, L: Florentín \& Arana 10 (CTES). 


\section{J. E. Florentín et al. - Taxonomia de Spermacoce (Rubiaceae)}

\section{Clave para identificar a Spermacoce eryngioides y especies afines}

1. Hojas subcoriáceas, de 5-12 × 2-2,5 mm; inflorescencias en ejes floríferos simples o con una división dicotómica apical, con glomérulo apical de mayor diámetro que los axilares; brácteas involucrales iguales o levemente más largas que el diámetro de los glomérulos; disco bilobado; cara ventral de la semilla plana, sin surcos longitudinales notorios.

S. ostenii

1'. Hojas cartáceas, de 13-75 × 1,5-26 mm; inflorescencias en ejes floríferos dicotómicos o pseudodicotómicos con divisiones hasta de quinto orden, con glomérulo apical más o menos del mismo diámetro que los axilares; brácteas involucrales 2-5 veces más largas que el diámetro de los glomérulos; disco entero o bipartido; cara ventral de la semilla con surco longitudinal más o menos notorio.

2. Hojas oblongas o elípticas, de 5-26 mm lat., base atenuada en un pseudopecíolo, con 5-6 nervios secundarios conspicuos; corola externamente pubescente, internamente con un anillo de pelos en la base de los lóbulos y escasos pelos ralos en la base del tubo; disco bipartido. S. hassleri

2'. Hojas lineares o angostamente elípticas, de 1,5-5 mm lat., sésiles, con 2-3 nervios secundarios inconspicuos; corola externamente glabra, internamente con franja densa de pelos en la mitad inferior de los lóbulos y un anillo de pelos en la base del tubo; disco entero. S. eryngioides

1. Spermacoce eryngioides (Cham. \& Schltdl.) Kuntze, Revis. Gen. Pl. 3(2): 123. 1898. Borreria eryngioides Cham. \& Schltdl., Linnaea 3: 316. 1828. Bigelovia eryngioides (Cham. \& Schltdl.) Hook. \& Arn., Bot. Misc. 3: 361. 1833. Tipo: Brasil. "Brasiliae meridionalis", F. Sellow s.n. (Lectotipo designado por Cabral et al. 2011: L 0057635!; isolectotipos E 00504647!, HAL 0098352!, W!).

Sufrútice, $10-30 \mathrm{~cm}$ alt., tallos tetrágonos, glabros. Hojas lineares o angostamente elípticas, sésiles, raramente pseudopecioladas, cartáceas, glabras, bordes revolutos, 13-23 × 1,5-5 mm, nervios secundarios inconspicuos; vaina estipular 1-4 mm long., glabra, con 6-7 lacinias, 1-4 mm long., linear-subuladas, glabras y desiguales. Ejes floríferos dicotómicos, con glomérulos más o menos iguales entre sí, brácteas involucrales 2-5 veces más largas que los glomérulos. Flores con hipanto 1-2,1 mm long., segmentos 0,9-1,8 mm long., pubescentes, lineares o angostamente triangulares, márgenes papilosos, con coléteres en los senos interlobulares; corola urceolada, 1,1-2,7 mm long., tubo 0,2-0,7 mm long., lóbulos 0,7-1,5 mm long., ápice agudo y levemente inflexo; mitad superior con papilas, internamente con franja de pelos en la mitad inferior de los lóbulos, tubo con pelos que siguen la vena media del lóbulo formando un segundo anillo de pelos laxos; estambres con filamentos 0,1-0,2 mm long., adnatos en la mitad superior del tubo corolino, anteras 0,25- 0,9 mm long., ovoides; granos de polen isopolares, radiosimétricos, 3-4-zonocolpados; pequeños $(\mathrm{P}=16-20 \mu \mathrm{m} ; \mathrm{E}=14-16 \mu \mathrm{m})$, subprolatos $(\mathrm{P} / \mathrm{E}=1,14-1,259)$, exina $2 \mu \mathrm{m}$ de espesor, tectadapsilada con espínulas ubicadas alrededor de los colpos; estilo 0,2-0,5 mm, sobrepasando al disco; estigma bilobado, ca. 0,14 mm long., con papilas unicelulares piriforme o lacrimiformes; disco entero, con papilas subcilíndricas, cutícula levemente estriada, estomas con células oclusivas de cutícula lisa, reborde estomático notable y apertura elíptica. Fruto subgloboso, 1,2-3,1 mm long., dehiscencia septicida, valvas dehiscentes. Semilla elipsoidales o subelipsoides, 1-1,5 x 0,4-0,7 $\mathrm{mm}$ long., cara ventral plana con surco longitudinal cubierto por el estrofíolo; exotesta retículo-foveada, células poligonales o de contorno circular, paredes anticlinales rectas y periclinales cóncavas.

Nombre vulgar: "Suspiro de ánimas".

Distribución y hábitat: Vive en Sudamérica y se distribuye en el centro-norte de Argentina, centrosur de Bolivia, sur de Brasil, Paraguay y sur de Uruguay (Fig. 1). Abundante en suelos modificados, arenosos, ladera de sierras, suelo rocoso, con sectores de tierra negra, plazas, bordes de camino, borde de bosques, campos y áreas cultivadas.

Fenología: Florece y fructifica todo el año.

Material adicional examinado. ARGENTINA. Prov. Corrientes: Dpto. Capital, Ruta 12, $1 \mathrm{~km}$ $\mathrm{N}$ del Arroyo, Sombrero, 4-I-1976, Schinini et al. 12329 (CTES). Dpto. San Roque, 16 km S de 


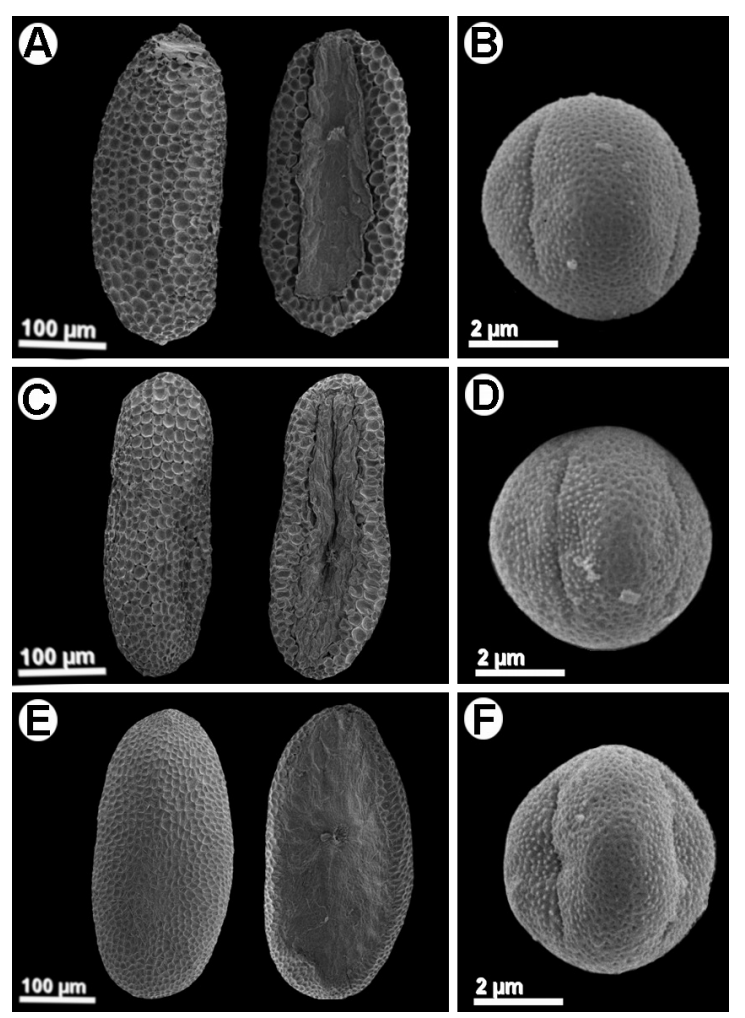

Fig. 4. Semillas y Polen. A-B. Spermacoce hassleri. A. Semillas B. Polen. C-D. S. eryngioides. C. Semillas. D. Polen. E-F. S. ostenii. E. Semillas F. Polen. A-B: Florentín \& Salas 9 (CTES); C-D: Judkevich \& Salas 57 (CTES); E-F: Florentín \& Arana 10 (CTES).

San Roque, Ruta 19, 7-IV-1970, Carnevali 2066 (CTES). Dpto. Ituzaingó, $17 \mathrm{~km} \mathrm{NW}$ de San Carlos, Rincón Chico, 11-XII-1984, Tressens et al. 2739 (CTES). Dpto. Concepción, $5 \mathrm{~km} \mathrm{SW} \mathrm{de}$ Santa Rosa, 29-III-1975, Arbo et al. 1023, (CTES). Dpto. San Cosme, Paso de la Patria, 1-X-1954, Carnevali 412 (CTES). Dpto. San Martín, La Cruz, cerro Susini, 15-II-1979, Schinini et al. 17209 (CTES). Dpto. Curuzú Cuatiá, ruta 119, 47 km S del acceso Mercedes, 20-II-1984, Tressens et al. 2228 (CTES). Dpto. Goya, camino de la Sociedad Rural, Aeroclub Goya, 19-II-1970, Carnevali 6279 (CTES). Dpto. Gral. Paz, Lomas de Vallejos, 29VIII-1973, Schinini et al. 7013 (CTES). Dpto. Mercedes, ruta 123, 103 m, 10-X-2008, Keller et al. 6397 (CTES). Dpto. San Miguel, 21 km S de Loreto, 7-III-1974, Schinini et al. 8253 (CTES). Dpto. Paso de los Libres, ruta 23, 2 km E del Río
Miriñay, 21-IX-1973, Schinini et al. 6267 (CTES). Dpto. Lavalle, ruta 12, $7 \mathrm{~km} \mathrm{~N}$ de empalme con ruta 120, 31-X-1974, Tressens et al. 657 (CTES). Dpto. San Luis del Palmar, Cañada Grande, ruta 8, 29-IX-1973, Tressens et al. 1429 (CTES). Dpto. Monte Caseros, ruta 14 entre Libertad y Pucheta, 29-IV-1987, Sáenz \& Morrone 306 (CTES). Dpto. Santo Tomé, $3 \mathrm{~km}$ SW de Gobernador Virasoro, 2-XII-1981, Tressens et al. 1446 (CTES). Dpto. Esquina, estancia La Victoria, Reserva Zenni, 8-X-2008, Keller et al. 6311(CTES). Prov. Chaco: Dpto. Bermejo, arroyo Cangui Chico, 22-IX-1967, Krapovickas \& Cristóbal 13169 (CTES). Dpto. General Güemes, paraje Costa Rica, 5 km E de 4 de Febrero, cauce de arroyo Guaycurú, 27-XI-1996, Krapovickas \& Schinini 46866 (CTES). Prov. Formosa: Dpto. Formosa, ruta 11, riacho Pilagá, 14-XI-1986, Cristóbal et al. 2116 (CTES). Dpto. Laishi, reserva ecológica El Bagual, San Francisco de Laishi, 30-IX-1998, Di Giacomo 389 (CTES). Dpto. Pilcomayo, Clorinda, 19-VII-1946, Morel 1018 (CTES). Dpto. Pilagas, reserva Guaicolek, 16-VII-2009, Keller et al. 7153 (CTES). Prov. Misiones: Dpto. San Pedro, Santa Teresa, 5-VII1949, Schwindt 1926 (CTES). Dpto. Candelaria, Loreto, reserva Yatay Poñi, 8-XII-2011, Miguel et al. 4 (CTES). Dpto. Concepción, sin localidad indicada, 15-IX-2008, Keller \& Franco 6053 (CTES). Prov. Salta: Dpto. Metán, Río Blanco, 23-IV-1988, Saenz et al. 577 (CTES). Prov. Santa Fe: Dpto. General Obligado, $17 \mathrm{~km}$ W de Villa Ana, 30-XI-1972, Quarin 745 (CTES). Prov. Tucumán: Dpto. Trancas, 23-IV-1988, Saenz et al. 543 (CTES). Prov. Córdoba: Dpto. Sobremonte, 4-V-1956, Hunziker 12084 (CTES). BOLIVIA. Dpto. Santa Cruz: Prov. Velasco, $63 \mathrm{~km} \mathrm{~S}$ de Rafael, camino a San Rafael, Serranía del Diablo, 4-IV-2006, Dematteis et al. 2222 (CTES). Dpto. Tarija: Prov. Arce, $63 \mathrm{~km}$ de Tarija hacia Bermejo, 24-I-1988, Ehrich 386 (CTES). BRASIL. Est. Mato Grosso: Santa Isabel, $13 \mathrm{~km}$ da rodovia Transpantaneira, km 117, direção ao Poconé, 22IX-1992, Prado 1648 (UEC). Est. Rio Grande do Sul: Morro Santa Teresa pr. Porto Alegre, 3-X-1949, Rambo 43698 (CTES); Serra Geral do Paraná, ca. 3 km S of São João da Aliança, 14-III-1971, Irwin et al. 31720 (UEC). Est. Goiás: rodovia Alto Paraíso de Goiás para Niquelândia, 26-IV-2009, Queiroz et al. 14322 (HUEFS). PARAGUAY. Dpto. Alto Paraguay: Puente, 28-XI-1988, Caballero Marmori 


\section{J. E. Florentín et al. - Taxonomia de Spermacoce (Rubiaceae)}

1457 (CTES). Dpto. Caaguazú: Guayaquí, 9-XI1993, Zardini \& Tellería 37218 (CTES). Dpto. Central: Esteros de Trinidad, 5-I-1951, Schwarz 11483 (CTES, LIL). Dpto. Concepción: estancia San Rafael, 140 km al E de Concepción, 21-III1991, Eliceche 122 (CTES). Dpto. Cordillera: San Bernardino, IX-1970, Schinini 2855 (CTES). Dpto. Misiones: Santiago, estancia La Soledad, ca. Isla Carpincho, 21-X-1967, Lourteig 2075 (CTES). Dpto. Paraguarí: Capilla Tenja, III-1969, Schinini 2818 (CTES). Dpto. Presidente Hayes: Villa Hayes, ruta 12, $5 \mathrm{~km}, 20-\mathrm{VI}-1975$, Krapovickas \& Schinini 28570 (CTES). URUGUAY. Dpto. Florida: Ruta 7 a $1 \mathrm{~km}$ de la estancia La Palma, $33^{\circ} 32^{\prime} 24^{\prime \prime} \mathrm{S}-55^{\circ}$ 13' 22” W, 30-XI-2001, Seijo et al. 2594 (CTES).

2. Spermacoce hassleri E. L. Cabral \& J. Florentín, nom. nov. $\equiv$ Borreria affinis DC., Prodr. 4: 546. 1830. Spermacoce affinis Pohl ex DC., Prodr. 4: 546. 1830, pro. syn., nom. inval. Borreria eryngioides var. affinis (DC.) K. Schum., en Martius, Fl. Bras. 6(6): 48. 1888. Tipo: Brasil. "Brasilia", Pohl s.n. (Holotipo $\mathrm{G}$ !; isotipo $\mathrm{M} !)$.

= Borreria assurgens var. longisepala Hassl., Repert. Spec. Nov. Regni Veg. 14: 167. 1915. Tipo: Paraguay. Cordillera, "ad ripas fluminis Piribebuy prope Emboscada”, V-1913, Hassler 12643 (Holotipo G!; isotipos BAF!, K 000470315 !, MO!).

= Borreria chacoensis Hassl., Repert. Spec. Nov. Regni Veg. 14: 167. 1915. Spermacoce chacoensis (Hassl.) Govaerts, World Checkl. Seed Pl. 2(1): 15. 1996. Tipo: Paraguay. Distrito Capital"Gran Chaco, in campis humidis Loma Clavel”, XI-1903, Hassler 2617 (Lectotipo, designado por Cabral \& Bacigalupo 1996: G!; isolectotipos K 000470324!, NY 00130916!).

= Borreria chacoensis var. glabrata Hassl., Repert. Spec. Nov. Regni Veg. 14: 168. 1915. Tipo: Paraguay. Boquerón-Presidente Hayes, "Gran Chaco, ad ripas fluminis Pilcomayo", VI-1906, Rojas 247 (Holotipo G!).

= Borreria diffusa DC., Prodr. 4: 544. 1830. Tipo: Brasil. "Brasilia", Pohl s.n. (Holotipo G!; isotipos K 00470199!, K 00470200!).

= Borreria lagurus S. Moore, Trans. Linn. Soc. London, Bot. 4: 383. 1895. Spermacoce lagurus (S. Moore) Govaerts, World Checkl. Seed Pl. 2(1): 16. 1996. Tipo: Brasil. "Crescit ad Santa Cruz, XI”, Mato Grosso, 1891, Moore 668 (Holotipo BM 000053653!).
= Borreria nectarifera Rusby, Mem. New York Bot. Gard. 7: 381. 1927. Tipo: Bolivia. Beni, Pampas near Lake Rogagua, 4-IV-1921, Rusby 1659 (Holotipo NY 00621887!).

Sufrútice $50-100 \mathrm{~cm}$ alt., tallos tetrágonos, glabros o pubescentes. Hojas oblongas o elípticas, pseudopecioladas, subcartáceas, pubescentes, bordes revolutos, escábridos, 15-75 x 5-26 mm, 5-6 nervios secundarios evidentes; vaina estipular 1-3,24 mm long., pubescente, con 6-7 lacinias, 1,4$3,8 \mathrm{~mm}$ long., linear-subuladas, glabras, desiguales. Eje florífero pseudodicotómicos, ampliamente ramificada, con glomérulos apicales de menor diámetro que los subapicales, brácteas involucrales 2-5 veces más largas que los glomérulos. Flores con hipanto 1,70-2 mm long., segmentos 1-2,4 mm long., pubescentes, angostamente triangulares o angostamente ovados, iguales o de mayor longitud que la corola, márgenes glabros, con coléteres interlobulares; corola urceolada, 1,8-2,9 mm long., tubo 0,2-0,9 mm long., lóbulos 1-2 mm long., ápice acuminado e inflexo, externamente pubescente, mitad superior de los lóbulos con papilas, internamente con anillo de pelos en la mitad inferior de los lóbulos y con algunos pelos ralos en la línea media de los lóbulos y tubo; estambres subsésiles, filamentos 0,04-0,4 mm long., adnatos a la mitad superior del tubo corolino, anteras de 0,42 $1 \mathrm{~mm}$ long., ovoides; granos de polen isopolares, radiosimétricos, 3-4-zonocolpados, pequeños $(\mathrm{P}=15-19 \mu \mathrm{m}, \mathrm{E}=13-15 \mu \mathrm{m})$, subprolatos $(\mathrm{P} /$ $\mathrm{E}=1,15-1,26)$; exina $2 \mu \mathrm{m}$ espesor, tectada-psilada con espínulas ubicadas a lo largo de los colpos; estilo 0,1-0,3 mm long., igual longitud que la altura del disco; estigma ca. 0,19 mm long., bilobado, con papilas unicelulares, piriformes o lacrimiformes; disco bipartido, con papilas subcilíndricas y cónicas, cutícula estriada, estomas con células oclusivas de cutícula lisa, reborde estomático notable y apertura elíptica. Fruto subgloboso, pubescente, 2,8-3,5 mm long., dehiscencia septicida, valvas dehiscentes; semilla elipsoidales o subelipsoides, 1-1,5 x 0,4-0,7 $\mathrm{mm}$, cara ventral con surco longitudinal amplio, cubierta por el estrofíolo; exotesta retículo-foveada, células poligonales o de contorno circular, paredes anticlinales rectas y periclinales cóncavas.

Distribución y hábitat: vive en el centro y norte de Argentina, en el centro-sur de Brasil, Bolivia y 
Paraguay (Fig. 1). Se encuentra frecuentemente en campos bajos o próximos a cursos de ríos, suelos inundables, palmares, bañados y pastizales.

\section{Fenología: Florece y fructifica todo el año.}

Material adicional examinado. ARGENTINA. Prov. Córdoba: Dpto. San Justo, entre Villa Concepción del Tío y El Tío, 6-XI-1952, Hunziker 10071 (CORD). Prov. Catamarca: Dpto. Ambato, $2 \mathrm{~km}$ NE del Bolsón, Finca del Cevil, 3-III1995, Saravia Toledo et al. 13253 (CTES). Dpto. Antofagasta de la Sierra, Cuesta de la Loma Larga, ruta 62, km 1421-1422, entre Singuil y Las Chacritas, 4-IX-1971, Hunziker et al. 21198 (CTES). Dpto. Santa Rosa, Bañado de Obanta, 3-XII-1944, Pierotti 99722 (CTES). Prov. Corrientes: Dpto. Capital, Riachuelo, 10-VII1980, Cabral 178 (CTES). Dpto. Curuzú Cuatiá, 15 km S de Curuzú Cuatiá, 10-VIII-1971, Carnevali 2602 (CTES). Dpto. Ituzaingó, 17 km NW de San Carlos, Rincón Chico, 5-III-1985, Tressens et al. 3124 (CTES). Dpto. Santo Tomé, ruta 40, 6 km SW de Colonia Garabí, arroyo Ciriaco, 12-XII-1984, Tressens et al. 2904 (CTES). Prov. Chaco: Dpto. Almirante Brown, $40 \mathrm{~km} \mathrm{~N}$ de Pampa del Infierno, Fachinal, 2-II-1978, Bordón 565 (CTES). Dpto. Bermejo, Isla Cerrito, 2-II-2002, Schinini 35799 (CTES). Dpto. San Fernando, Isla Soto, 12-IX1974, Burkart 31114 (CTES). Prov. Formosa: Dpto. Matacos, Ingeniero Juárez, 1-VII-1962, Bordón 8 (CTES). Dpto. Pilcomayo, ruta 86, $50 \mathrm{~km}$ al W del Arroyo Porteño, 10-II-1981, Valla et al. 17574 (CTES). Prov. Jujuy: Dpto. Capital, 2 km S de Palpalá, 29-III-1977, Krapovickas \& Schinini 30670 (CTES). Dpto. El Carmen, Pampa Blanca, 5-XI-1987, Ahumada \& Castellón 5459 (CTES). Dpto. Ledesma, Parque Nacional Calilegua, 9-VII1995, Ahumada \& Castellón 7260 (CTES). Dpto. Santa Bárbara, Palma Sola, 19-I-2012, Sato et al. 268 (CTES). Dpto. San Pedro, Palmera, 14-VII1949, Reales 1914 (CTES). Prov. Misiones: Dpto. Candelaria, San Juan, 1-X-1952, Montes 15507 (CTES). Dpto. Cainguás, Campito 5, 17-III-2000, Biganzoli et al. 935 (CTES). Dpto. San Ignacio, Teyucuaré, 21-III-1951, Montes 15148 (CTES). Prov. Salta: Dpto. Iruya, Isla de Cañas, 12-III2007, Cabral et al 764 (CTES). General José de San Martín, Finca Yavere, RN 81, a 10 km E de Senda Hachada, 265 m, 14-II-1991, Novara 10173
(CTES). Dpto. La Capital, La Merced, 16-II-1949, Legname 478 (CTES). Dpto. Metán, ruta 5, ca. 5 $\mathrm{km}$ de la ruta 9, 27-VI-2007, Souza 8021 (CTES). Dpto. Orán, 28 km N de Orán, Quebrada del Anta Muerta, 12-VII-1972, Maruñak et al. 479 (CTES). Dpto. Rosario de Lerma, 26-III-1977, Krapovickas \& Schinini 3055 (CTES). Prov. Santa Fe: Dpto. General Obligado, Los Laureles, 23-XI-1986, Blanchoud 2252 (CTES). Dpto. San Cristóbal, Hersilia, 18-XI-1946, Balegno 575 (CTES). Prov. Santiago del Estero: Dpto. Guasayán, Quebrada de Tazana, 3-IV-1944, Pierotti 99456 (CTES). Dpto. Capital, Cevillar, 13-III-1944, Pierotti 81561 (CTES). BOLIVIA. Dpto. Santa Cruz: Prov. Andrés Ibañez, N Santa Cruz de la Sierra, 1744’30”S, 63¹2’00”W, 7-XI-1990, Nee 39716 (CTES); ídem, 13-II-1995, Abbott 16176 (CTES). Prov. Chiquitos, $5 \mathrm{~km}$ E de San José camino a Robore, 6-IV-2006, Dematteis et al. 2253 (CTES). Prov. Cordillera, Charaguas 18 km E, 31-III-1993, Martínez 12463 (CTES). Prov. Ñuflo de Chávez, $25 \mathrm{~km} \mathrm{SW}$ de San Javier, camino a San Ramón, 31-III-2006, Dematteis et al. 2043 (CTES). Prov. Velasco, $63 \mathrm{~km}$ $\mathrm{S}$ de San Rafael, camino a San José, Serranía del Diablo, 4-IV-2006, Dematteis et al. 2202 (CTES). Prov. Valle Grande, $5 \mathrm{~km} \mathrm{~N}$ de El Trigal, 9-IV-2009, Meza Torres et al. 1241 (CTES). Dpto. Beni: Prov. Vaca Diez, Guayaramerín, 16-IV-1979, Krapovickas \& Schinini 35051 (CTES). Prov. Moxos, camino a San Ignacio de Moxos a Santa Ana de Yucuma, km 30, 26-VIII-2009, Parada et al. 1574 (CTES). Dpto. Tarija, $19 \mathrm{~km} \mathrm{~N}$ de Camatindi, camino a Boyuibe, 29-III-2006, Dematteis et al. 1947 (CTES). Dpto. Tarija: Villa Montes, $140 \mathrm{~km}$ de Tarija, 24-V1971, Krapovickas et al. 19176 (CTES). Prov. Arce, 13-III-1974, Turpe 4752 (CTES). BRASIL. Est. Alagoas: Piranhas, $16 \mathrm{~km}$ após a Piranhas, em direção à Aragarças, 16-V-2005, Souza 1208 (CTES). Est. Distrito Federal: Brasilia, 18-III1999, Pereira et al. 4086 (CTES). Est. Mato Grosso do Sul: Corumbá, 12-II-1981, Almeida 36 (UEC); Município de Pocone Ipiranga, 6-XII-1991, Schess 1248 (CTES); Fazenda Santa Isabel, ca. 13 km à direita da rodovia Transpantaneira Poconé - Porto Jofre, 22-X-1992, Prado 3048 (UEC); Rio Perdido, 23-V-2002, Hatschbach et al. 73352 (CTES); Aldeia indígena, 19-III-2003, Hatschbach et al. 74923 (CTES). Est. Paraná: Curitiba, Fazenda das Acacias, Rio das Contas, 3-I-2002, Carneiro 1257 (CTES). Est. Rio Grande do Sul: Montenegro, 


\section{J. E. Florentín et al. - Taxonomia de Spermacoce (Rubiaceae)}

17-VIII-1949, Rambo 42974 (CTES). Est. São Paulo: Sítio Santana, Oswaldo Cruz, 6-VIII-1996, Souza \& Souza 11440 (ESA). PARAGUAY. Dpto. Alto Paraguay: sin localidad, 9-II-2002, Zardini \& Rivas 58333 (CTES). Dpto. Amambay: $8 \mathrm{~km} \mathrm{~W}$ de Bella Vista, camino a San Carlos, 15-XII-1999, Ferrucci et al. 1522 (CTES). Dpto. Boquerón: Pozo Colorado, 4-III-1980, Caballero 630 (CTES). Dpto. Central: Tavarory, 9-XII-1991, Zardini \& Telleria 29225 (CTES). Dpto. Itapúa: sin localidad, 9-XII2000, Zardini \& Gamarra 59753 (CTES). Dpto. Paraguari: sin localidad, 27-VI-1992, Zardini \& Telleria 30107(CTES). Dpto. Presidente Hayes: Estancia Salazar, 12-XI-1993, Pérez et al. 2874 (CTES); 78 km E de Pozo Colorado, 19-XII-1987, Schinini \& Palacio 25898 (CTES).

3. Spermacoce ostenii (Standl.) E. L. Cabral \& J. Florentín, nov. comb. Borreria ostenii Standl., Ostenia 119, t. 19. 1993. Borreria eryngioides var. ostenii (Standl.) E. L. Cabral \& Bacigalupo, Opera Bot. Belg. 7: 317. 1996. Tipo: Argentina. Córdoba, Sierra Chica, La Falda, 24-IV-1917, Osten 10631 (Holotipo MVM!; isotipos CORD 00004455!, F!).

Sufrútice 10-30 cm alt., tallos tetrágonos, glabros. Hojas elípticas o lineares, sésiles o cortamente pseudopecioladas, subcoriáceas, glabras, bordes revolutos engrosados, 4-10 × 1-2 mm, nervios secundarios inconspicuos, con braquiblastos foliosos axilares; vaina estipular 1-5 mm long., glabra, con 6-7 lacinias, 1,2-4 mm long., lineares-subuladas, glabras o pubérulas, desiguales. Ejes floríferos simples o rara vez divididos en el ápice con glomérulos 6-10 mm lat., el terminal de mayor tamaño que los inferiores; brácteas involucrales 4-7,5 mm long., iguales o levemente mayores que el glomérulo. Flores con hipanto 1,1-2,34 mm long., segmentos 1,2-2,7 mm long., angostamente triangulares, de igual o mayor longitud que la corola, glabros, con coléteres interlobulares; corola urceolada, 1,7-3,5 mm long., tubo 0,43-1,35 mm long., lóbulos 1,22,3 mm long., angostamente ovados, ápice agudo e inflexo, mitad superior con papilas, externamente papilosa, internamente con franja densa de pelos en la mitad inferior de los lóbulos, en la línea media del lóbulo corolino y hasta la base del tubo formando un anillo; estambres subsésiles, filamento 0,1-0,2 mm long., adnatos a la mitad superior del tubo corolino, anteras 0,7- 0,9 mm long., oblongas; granos de polen isopolares, radiosimétricos, 3-4-zonocolpados, pequeños $(\mathrm{P}=18-20 \mu \mathrm{m}, \mathrm{E}=15-17 \mu \mathrm{m})$, subprolatos $(\mathrm{P} / \mathrm{E}=1,12-1,17)$; exina $2 \mu \mathrm{m}$ espesor, tectadapsilada, con espínulas ubicadas alrededor de los colpos; estilo 0,1-0,4 mm long., incluido en el disco; estigma ca. 0,23 mm long., bilobado, con papilas unicelulares, piriformes o lacriformes; disco bilobado, con papilas cónicas de cutícula estriada, estomas con células oclusivas de cutícula lisa, reborde estomático notable y apertura elíptica. Frutos subgloboso, pubescente, 2,9-4,1 mm long., dehiscencia septicida, valvas dehiscentes; semillas elipsoidales, $1-1,4 \times 0,17-0,71 \mathrm{~mm}$, cara ventral plana cubierta totalmente por el estrofíolo, sin surco evidente; exotesta retículo foveada, células de contorno circular o hexagonal, paredes anticlinales rectas y paredes periclinales cóncavas.

Distribución y hábitat: Endémica de Argentina, vive en un área muy restringida de las sierras de Córdoba y San Luis, entre 800-1300 m de altura (Fig. 1). Es escasa y habita en afloramientos rocosos y suelos secos.

Fenología: Florece de septiembre a marzo y fructifica de abril a junio.

Material adicional examinado. ARGENTINA. Prov. Córdoba: Dpto. Calamuchita, Villa Rumipal, 17-I-1951, de la Sota 4019 (CORD, CTES). Dpto. Pocho, a mitad de camino entre Las Palmas y La Mudana, 16-II-1952, Hunziker 9784 (CORD). Dpto. Punilla, Sierra Grande (Falda E), Cerro Blanco, inmediaciones de Tanti, 7-IV-1973, Subils \& Anton 1676 (CORD); Sierra Chica (Falda O), observatorio de Bosque Alegre, 25-I-1955, Hunziker 10612 (CORD), ídem, al sur de Valle Hermoso, en Vaquerías, subiendo al cerro de la Cruz, $900 \mathrm{~m}$, 12-III-1977, Hunziker 22961 (CORD); Los Cocos, 24-I-1947, Villafañe 527 (CTES); Thea, 30-I-1947, Villafañe 664 (CTES); La Falda, 7-II-1947, Villafañe 754 (CTES); Sierra Chica, Cerro Uritorco, 14-IV1915, Hosseus 13 (CTES); Dpto. Río Cuarto, Achira, Monte Guazú, 1185 m, 8-III-2010, Cantero 6294 (CORD); Alpa Corral, 24-VI-1999, Ceballos 3332 (CRC); ídem, 20-VII-1996, Correa 2571 (CRC); Cerro Azul, 922 m, 2009, Cantero 5460 (CORD); Las Guindas, 27-III-2000, Ceballos 3627 (CRC). Dpto. Santa María, La Ochoa, cerro Pelado, 11III-1951, Hunziker 8982 (CORD). Dpto. Tulumba, 
Cerros de Sauce Puncú, entre Deán Funes e Inti Huasi, 28-IV-1951, Hunziker 9206 (CORD). Prov. San Luis: Dpto. Coronel Pringles, Trapiche, II-1973, Boffa s.n. (LP); Dpto. Pedernera, Sierra El Morro, 1200 m, 27-XII-1977, Anderson et al. 3434 (CORD).

Tratadas bajo Borreria, B. eryngioides var. eryngioides, Borreria eryngioides var. affinis y Borreria eryngioides var. ostenii fueron agrupadas bajo una misma especie por Cabral \& Bacigalupo (1996), por tener estambres y estigma inclusos, corola urceolada de mayor longitud que los segmentos del cáliz. Algunos de los caracteres aquí señalados para separar las especies fueron utilizadas por las autoras para diferenciar dichas variedades. Sin embargo, en el presente trabajo se suman otros caracteres provenientes del indumento interno de la corola, forma del disco nectarífero, cara ventral de la semilla, células de la exotesta, y no menos importante información ecológica y de su distribución, que soportan sus rehabilitaciones a especie.

\section{Transferencia de Borreria secc. Pseudodiodia a Spermacoce}

En cuanto a la transferencia de Borreria secc. Pseudodiodia, prácticamente todas las características diagnósticas del taxón son compartidas con el género Spermacoce como fuera definido por Cabral et al. (2010). La única diferencia resaltable responde a la dehiscencia del fruto, i.e. cápsulas con ambas valvas dehiscentes (vs. cápsulas con una valva dehiscente y la otra indehiscente en Spermacoce, sensu Cabral et al. 2010).

Pire (1996) estudió la morfología polínica de la mayoría de las especies de Borreria secc. Pseudodiodia e incluyó a todas las especies de este grupo dentro del Tipo I, el cual se caracteriza por presentar granos 3-7-colporados, con ectoaperturas de colpos largos y estrechos, endoaperturas lalongadas o unidas lateralmente, subprolatos o prolato-esperoidales, pequeños $(\mathrm{P}=14-17 \mu \mathrm{m})$, tectados, foveolados o raramente tectados perforados y parcialmente espinulados (espínulas localizadas alrededor de las aperturas). El tipo polínico I, es compartido con Spermacoce, por lo cual se toma como un fuerte soporte para la reubicación de la sección en estudio.

Por su parte, Dessein et al. (2002) al estudiar especies africanas de Spermacoce utilizaron los mismos tipos polínicos que Pire (1996) y consideraron en éste a tres especies: Spermacoce mauritiana Gideon [= Spermacoce exilis (L. O. Williams) C. D. Adams], S. natalensis Hochst. y $S$. tenuior. La primera especie es la única que habita en América que pertenece a Borreria secc. Pseudodiodia, mientras que la última es la especie tipo de Spermacoce.

Los estudios filogenéticos son realmente escasos y el único trabajo que incluye simultáneamente a especies de ambos taxones fue llevado a cabo por Salas et al. (2015). Si bien las especies de Spermacoce y Borreria secc. Pseudodiodia están escasamente representadas, se observa que las especies analizadas de Pseudodiodia (i.e. S. eryngioides, S. incognita y S. prostrata, mencionadas bajo Borreria) caen anidadas entre especies de Spermacoce de Australia y de Spermacoce s.str. (todas americanas, incluyendo la especie tipo). Por lo expuesto, se considera que existe suficiente soporte para considerar a las especies de Borreria secc. Pseudodiodia en Spermacoce.

Cabral \& Bacigalupo (1996) y Cabral et al. (2006) incluyeron 10 especies en Borreria secc. Pseudodiodia, de las cuales sólo las especies brasileñas fueron consideradas en Spermacoce (Cabral \& Salas, 2015), por lo que se hace necesario incluir una lista actualizada de dichas especies.

La lista fue elaborada en base a lo siguiente: a) solamente se listan especies que fueron consideradas en Borreria secc. Pseudodiodia y que por sus características morfológicas pertenecen a Spermacoce; b) algunas especies listadas fueron sinonimizadas por Cabral \& Bacigalupo (1996) o Cabral et al. (2006), sin embargo en este trabajo se los consideran como válidas a nivel de especie [e.g. Spermacoce exilis, S. ovalifolia (M. Martens \& Galeotti) Hemsl., S. simplicicaulis (K. Schum. ex Sucre) Govaerts]; c) la lista no incluye nuevas combinaciones dado que las especies ya fueron previamente consideradas bajo Spermacoce por otros autores; y d) de las especies tratadas previamente en este trabajo, se incluyen sólo los nombres para no repetir información.

Lista de las especies de Borreria secc. Pseudodiodia consideradas aquí en Spermacoce

1. Spermacoce decipiens (K. Schum.) Kuntze, Revis. Gen. Pl. 3: 123. 1898. Borreria decipiens K. Schum., in Martius, Fl. Bras. 6(6): 57. 1889. Tipo: 


\section{J. E. Florentín et al. - Taxonomia de Spermacoce (Rubiaceae)}

Brasil. "Provincia Piauhy" [Probablemente Piauí], Gardner 2193 (Holotipo B [destruido], foto F OBN 000874!], isotipos BM 000053645!, F 875671!, G!, IPA!, K 000016113!, K 000016114!, L 0000140!, P 02285142!, P02285143!, P02285144!, RB 41884!, S 05-1624!, W 0028703!, W18890113597!).

2. Spermacoce dispersa (Hook. f.) Kuntze, Revis. Gen. Pl. 3(3): 123. 1898. Borreria dispersa Hook. f., in Hooker, Trans. Linn. Soc. London 20: 217. 1847. Tipo: Ecuador. Galápagos, "Charles and James islands", 1835, Darwin s. n. (Lectotipo designado por Wiggins \& Porter, 1971: CGE 00337!; isolectotipos: K 000470284!, K 000470285!, K 000470287!).

3. Spermacoce eryngioides (Cham. \& Schltdl.) Kuntze.

4. Spermacoce exilis (L. O. Williams) C. D. Adams, in Burger \& Taylor, Fieldiana, Bot., n.s. 33: 316, f. 5. 1993. Borreria exilis L. O. Williams, Phytologia 28: 227. 1974. Borreria gracilis L. O. Williams, Phytologia 26: 487. 1973, nom. illeg. Tipo: Costa Rica. Cocos Islands, Wafer Bay, 28-VI-1932, Howell 10178 (Holotipo F!).

5. Spermacoce gracillima (DC.) Delprete, Rev. Biol. Neotrop. 3: 72. 2006. Borreria gracillima DC., Prodr. 4: 543. 1830. Tipo: Brasil. "Brasilia", 1828, Pohl s.n. (Holotipo G-DC!).

6. Spermacoce hassleri E. L. Cabral \& J. Florentín.

7. Spermacoce incognita (E. L. Cabral) Delprete, J. Bot. Res. Inst. Texas 1: 1025. 2007. Borreria incognita E. L. Cabral, Opera Bot. Belg 7: 322, f. 8. 1996. Tipo: Brasil. Goiás, Campo Alegre, Rod. BR-050, km 222, 6-XI-1994, Hatschbach \& Silva 59881 (Holotipo MBM!; isotipos BHCB 00000402!, BR 05305278!, C 10018072!, CTES 0013511!, G 00389686!, G 00389687!, NY 00074088!, MO 00260420!, SI 003179!, SI 003547!, SPF 00109332!).

8. Spermacoce neotenuis Govaerts, World Checkl. Seed Pl. 2(1): 18. 1996. Borreria tenuis DC., Prodr. 4: 543. 1830. Tipo: Brasil. "Brasilia", 1828, Pohl s.n. (Holotipo G-DC!).

9. Spermacoce ocymoides Burm. f., Fl. Indica 34, pl. 13, f. 1. 1768. Borreria ocymoides (Burm. f.) DC., Prodr. 4: 544. 1830. Tardavel ocymoides (Burm. f.) Hiern, Cat. Afr. Pl. Welw. 2: 504. 1898. Tipo: Indonesia. "Sajor Babi Javanis" [Probablemente Java], Kleinhof s.n. (Holotipo G!).

10. Spermacoce ostenii (Standl.) E. L. Cabral. \& J. Florentín.
11. Spermacoce ovalifolia (M. Martens \& Galeotti) Hems1., Biol. Cent.-Amer., Bot. 2: 59. 1881. Borreria ovalifolia M. Martens \& Galeotti, Bull. Acad. Roy. Sci. Bruxelles 11: 129-130. 1844. Tipo: México. Veracruz, Colonia de Mirador, 900 m, II- 1840, Galeotti 2606 (Holotipo BR 05325993!, isotipos BR 05325665!, K 00470223!).

12. Spermacoce prostrata Aubl., Hist. Pl. Guiane 1: 58, t. 20, f. 3. 1775. Tipo: Guayana Francesa. "Ad ripas fluviorum" and "sur le bord des rivieres", [1762-1764], Aublet s.n. (Lectotipo designado por Lanjouw \& Uittien 1940: P-JJR 3: 118E!). Una discusión acerca del tipo de esta especie se presenta en Delprete (2015).

13. Spermacoce reflexa (Kirkbr.) Govaerts, World Checkl. Seed Pl. 2: 18. 1996. Borreria reflexa Kirkbr., Acta Amazon. 10: 112. 1980. Tipo: Brasil. Bahia, Serra, $22 \mathrm{~km} \mathrm{~W}$ of Barreiras, ca. 620 m, 2-III-1972, Anderson et al. 36482 (Holotipo UB!; isotipos F 0068545F!, MO 00797238!, NY!, US!).

14. Spermacoce simplicicaulis (K. Schum. ex Sucre) Govaerts, World Checkl. Seed Pl. 2: 19. 1996. Borreria simplicicaulis K. Schum. ex Sucre, Rodriguésia 26: 253-254, fig. 3, 4. 1971. Tipo: Brasil. Goiás, fazenda do Cipó, perto de Itaquira, 3-II-1895, Glaziou 21514 (Holotipo R 00009976!; isotipos BR 05305612!, P 02285133!, P 02285134!, P 02285135!).

15. Spermacoce suberecta (Hook. f.) Kuntze, Revis. Gen. Pl. 3: 123. 1898. Spermacoce suberecta (Hook. f.) Fosberg, Phytologia 62: 183. 1987, comb. superfl. Borreria suberecta Hook. f., Trans. Linn. Soc. London 20: 217. 1847. Tipo: Ecuador. Galápagos, “Albemarle island" (actualmente isla Isabela), Macrae s.n. (Lectotipo aquí designado: K 000470282!, isolectotipo K 000470279 !). En la base de datos J-STOR aparecen tres ejemplares asociados al nombre de esta especie, depositados en $\mathrm{K}$, y todos poseen el sello del Herbario Hookerianum. Sin embargo uno de estos ejemplares corresponde a una colección de Drawin, y sólo dos colecciones son de Macrae. Se selecciona como lectotipo al material K 000470282 por ser un ejemplar representado por dos grandes ramas que cubren casi toda la cartulina y además tiene ilustraciones de los caracteres diagnósticos, realizados probablemente por el autor de la especie. 
16. Spermacoce tocantinsiana (E. L. Cabral \& Bacigalupo) Delprete., J. Bot. Res. Inst. Texas 1: 1028. 2007. Borreria tocantinsiana E. L.Cabral \& Bacigalupo, Kew Bull. 59: 284, f. 5. 2004. Tipo: Brasil. Tocantins, Conceição do Tocantins, fazenda Cartão e Vista, 11-V-2000, Hatschbach et al. 70928 (Holotipo MBM!; isotipos CTES 0013514!, RB 00543481!, SI 095276!).

\section{Agradecimientos}

El primer autor agradece a los Dres. Marcelo Arana y Antonia Oggero por la ayuda en las tareas de campo realizadas en las Sierras de Córdoba, y al Consejo Interuniversitario Nacional (CIN) por la beca para desarrollar este estudio. Los autores agradecen a los editores y a los revisores por la lectura crítica del manuscrito.

\section{Bibliografía}

BACIGALUPO, N. M. 1972. Observaciones sobre algunas especies de los géneros Spermacoce L. y Spermacoceodes O. K. (Rubiaceae). Darwiniana 17: 341-357.

BACIGALUPO, N. M \& E. L CABRAL. 1996. Infrageneric classification of Borreria (Rubiaceae Spermacoceae) on the basis of American species. Opera Bot. Belg. 7: 297-308.

CABRAL, E. L. \& N. M. BACIGALUPO. 1996. Revision of Borreria sect. Pseudodiodia. Opera Bot. Belg. 7: 309-328.

CABRAL, E. L. \& R. SALAS. 2015. Spermacoce. En: Lista de Espécies da Flora do Brasil. Jardim Botânico do Rio de Janeiro. Disponible en: http://floradobrasil. jbrj.gov.br/jabot/floradobrasil/FB14299. [Acceso: 3 Octubre 2015].

CABRAL, E. L., A. A. CABAÑA FADER \& N. M. BACIGALUPO. 2010. A new species of Spermacoce s. str. (Spermacoceae, Rubiaceae) from Eastern Brazil. Pl. Ecol. Evol. 143: 233-238.

CABRAL, E. L., E. MARI \& S.M. PIRE. 2006. Borreria secc. Pseudodiodia (Rubiaceae), aportes taxonómicos y palinológicos. Bonplandia 15: 79-90.

DESSEIN, S., S. HUYSMANS, E. ROBBRECHT \& E. SMETS. 2002. Pollen of African Spermacoce species (Rubiaceae). Morphology and evolutionary aspects. Grana 41: 69-.89.
DELPRETE, P. G. 2007. New combination and new synonymies in the genus Spermacoce (Rubiaceae) for the Flora of Goiás and Tocantins (Brazil) and the Flora of The Guianas. J. Bot. Res. Inst. Texas 1: 1023-1030.

DELPRETE, P. G. 2015. Typification and etymology of Aublet's Rubiaceae names. Taxon 64: 595-624.

ERDTMAN, G. 1966. Pollen morphology and Plant Taxonomy Angiosperm. An introduction to Palynology I. Hatner Publishing Company, New York and London. GOVAERTS, R. 1996. World checklist of seed plants. Vol. 2, part 1. Continental Publishing, Antwerp, Belgium.

HIJMANS, R. J. 2013. DIVA-GIS, a geographic information system for the analysis of biodiversity data. Versión 7.5. Disponible en: http:// www.diva. gis.org [Acceso: 20 Marzo 2015).

KAREHED, J., I. GROENINCKX, S. DESSEIN, T. J. MOTLEY \& B. BREMER. 2008. The phylogenetics utility of chloroplast and nuclear DNA markers and the phylogeny of the Rubiaceae tribe Spermacoceae. Molec. Phylogenet. Evol. 49: 843-866.

LANJOUW, J. \& H. UITTIEN. 1940. Un nouvel herbier de Fusée Aublet découvert en France. Recueil Trav. Bot. Néerl. 37: 158.

MORRONE, J. J. 2014. Biogeographical regionalisation of the Neotropical region. Zootaxa 3782: 1-110.

PIRE, S. M. 1996. Palynological study of American species of Borreria (Rubiaceae -Spermacoceae). Opera Bot. Belg. 7: 413-423.

PUNT, W., P. P. HOEN, S. BLACKMORE, S. NILSSON \& A. LE THOMAS. 2007. Glossary of pollen and spore terminology. Rev. Palaeobot. Palynol. 143: $1-81$.

SALAS R. M., D. SOTO \& E. L. CABRAL. 2011. Dos especies nuevas de Borreria (Rubiaceae) y observaciones taxonómicas en Rubiaceae de Bolivia. Brittonia 63: 286-294.

SALAS, R. M., E. L. CABRAL, P. L. VIANA, S. DESSEIN \& S. JANSSENS. 2015. Carajasia (Rubiaceae), a new and endangered genus from the Carajás mountain range, Pará, Brazil. En: Delprete, P.G. \& S. Dessein (eds.), Festschrift volume dedicated to Timothy Motley (1966-2013). Phytotaxa 206: 14-29.

WIGGINS, I. L. \& D. M. PORTER. 1971. Flora of the Galápagos Islands. 422. Stanford University Press, Stanford.

Recibido el 24 de noviembre de 2015, aceptado el 22 de junio de 2016. 